\title{
Hemispheric asymmetries in categorical judgments of direction versus coordinate judgments of velocity of motion
}

\author{
STEPHEN D. CHRISTMAN \\ University of Toledo, Toledo, Ohio
}

\begin{abstract}
Three experiments on visual field differences in motion perception are reported. Experiment $1 \mathrm{em}-$ ployed circular stimuli that grew or shrank either quickly or slowly. Experiments 2 and 3 employed circles that moved upward or downward either quickly or slowly. Judgments based on categorical equivalence classes (i.e., grow/shrink, upward/downward) generally yielded small and nonsignificant right visual field advantages. Judgments based on the precise coordinates of motion (i.e., quickly/slowly) yielded significant left visual field advantages across all three experiments. Results are interpreted in light of Kosslyn's (1987) model of hemispheric differences in the processing of categorical versus coordinate spatial relations.
\end{abstract}

Research on hemispheric asymmetry has revealed numerous differences in how the left and right hemispheres (LH and $\mathrm{RH}$ ) process perceptual information, including spatial and temporal frequency processing, local/global processing, visual search, and the processing of different types of spatial relations (see Christman, 1997a). However, little attention has been paid to the question of hemispheric differences in motion perception. This reflects, in part, difficulties in creating and presenting dynamic stimuli on the desktop computers that are prevalent in psychology laboratories. To a lesser extent, this also reflects limitations in the amount of information that can be presented within the 200-msec (or less) exposure duration required by laterality studies. However, advances in software and hardware over the past decade have made the construction and presentation of dynamic stimuli available even to researchers without sophisticated programming skills or access to specialized hardware.

The few studies addressing hemispheric differences in motion processing reveal a mixed pattern of results (Bosworth \& Dobkins, 1999; Calvert, 1988; Dieterich, Bucher, Seelos, \& Brandt, 1998; Eals, 1987; Hollants-Gilhuijs, De Munck, Kubova, Royen, \& Spekreijse, 2000; HollantsGilhuijs, Ruijter, \& Spekreijse, 1998; Kostelyanets, Kamenkovich, \& Sharaev, 1992; Regan \& Vincent, 1995; Smith \& Hammond, 1986). In general, most of these studies report nonsignificant visual field differences. However, firm conclusions about hemispheric effects are limited by factors such as a lack of control over subject

The author thanks Melissa Feagin and Caroline Brackett for assistance in collecting data. The author also thanks Joseph Hellige and Karen Dobkins for helpful comments on an earlier version of the manuscript. Correspondence concerning this article should be addressed to S. D. Christman, Department of Psychology, University of Toledo, Toledo, OH 43606-3390 (e-mail: schrist2@uoft02.utoledo.edu). handedness, use of monocular viewing (which confounds hemiretinal and visual field effects), and use of simple detection tasks (which generally do not yield hemispheric differences; e.g., Christman, 1997b; Kitterle, Christman, \& Hellige, 1990). Although these studies indicate no absolute visual field asymmetry in motion perception, they do not rule out the possibility that different types of motion judgment may be carried out more efficiently in one or the other hemisphere.

A promising candidate for addressing potential hemispheric differences in motion processing derives from Kosslyn's (1987) model of how spatial relations are computed by the visual system. Kosslyn hypothesized that the visual system must extract two distinct types of spatial relations. First, there are categorical relations, in which discriminable differences in the distance between two objects are collapsed over, leading to an equivalence class; the relations above versus below are examples of categorical relations. Thus, the categorical representations of two stimuli that are both above a third stimulus but differ in distance from that third stimulus would be treated equivalently. The second type of spatial relation involves coordinate representations, in which the precise metric distance between objects is explicitly preserved. Thus, the coordinate representations of the two stimuli in the preceding example would involve discrete and discriminable representations.

Kosslyn's (1987) model addressed the neural substrates for categorical versus coordinate representations of spatial relations. The model hypothesizes that the computation of categorical versus coordinate representations is performed more efficiently in the left than in the right cerebral hemispheres (LH vs. RH), respectively. This prediction has been confirmed by various behavioral tests. For example, studies (e.g., Hellige \& Michimata, 1989; Kosslyn et al., 1989; Niebauer \& Christman, 
1998) have presented observers with stimuli in which a dot is presented at 1 of 12 possible locations relative to a line; 6 locations are above the line, and 6 are below. When subjects are asked to judge whether the dot is above or below the line (a categorical relation), LH advantages are typically obtained. Conversely, when subjects are asked to indicate whether the dot was near to or far from the line (a coordinate relation), RH advantages are typically obtained. This hypothesis of distinct neural substrates for spatial relation processing has also received indirect support from computational studies demonstrating that categorical and coordinate spatial relations are best computed by using qualitatively dissimilar networks (Kosslyn, Chabris, Marsolek, \& Koenig, 1992).

Kosslyn (1987) related the RH coordinate processing advantage to demands of spatial navigation, for which $\mathrm{RH}$ processing has been specifically implicated (e.g., De Renzi, 1982): “[A] coordinate representation . . . is especially useful for navigation, in which one needs to know where an obstacle is actually located, not just that is against a wall or next to some objects" (Kosslyn, 1987, pp. 153-154). Navigating through space involves dynamic changes in the visual input; yet, all tests to date of the categorical/coordinate framework have employed static displays. Given the important role of dynamic, moving visual input in spatial navigation, it seems appropriate to subject Kosslyn's hypothesis to a test involving stimuli that change size or position over time. That is, Kosslyn's model explicitly deals with the spatial relations between two simultaneously present stimuli, whereas the present paper proposes that this model can be extended to the spatial relations over time between successive positions of a single moving stimulus.

Accordingly, the purpose of the present paper is to present three experiments involving categorical and coordinate judgments of dynamic visual input. Experiment 1 utilized circles that either quickly or slowly grew or shrank in size, whereas Experiments 2 and 3 used circles that quickly or slowly moved upward or downward. Judgments of the direction of motion (i.e., grow/shrink, upward/downward) were hypothesized to reflect categorical processing, on the basis of the analogy with the above/below judgments used with the line-dot stimuli discussed above, whereas judgments of the velocity of motion were hypothesized to reflect coordinate processing.

An effective rubric for distinguishingbetween equivalenceclass-based categorical judgments and more precise, differentiating coordinate judgments is that, when comparing two objects that share a categorical judgment of upward motion (or are above a third object), it is not meaningful to refer to one object as moving "more upward" than the other (or that one object is "more above" the line). For stimuli sharing a common coordinate judgment, however, we can still distinguish between them by noting that although both objects may be moving fast (or are located near a third object), one object is nonetheless moving faster than the other (or is located nearer a third object). Categorical judgments of direction of motion (e.g., grow/shrink, upward/downward) were thus hypothesized to yield LH advantages, whereas coordinate judgments of velocity of motion (e.g., quick/slow) were hypothesized to yield $\mathrm{RH}$ advantages.

\section{EXPERIMENT 1}

\section{Method}

Subjects. Sixteen right-handed subjects with no left-handed relatives in their immediate family participated in order to receive extra credit in an introductory psychology course. Handedness was assessed via use of the Edinburgh Handedness Inventory (Oldfield, 1971).

Apparatus and Procedure. Stimuli were generated and presented on a Macintosh Quadra 660AV computer with the monitor set at a refresh rate of $67 \mathrm{~Hz}$. Stimuli consisted of QuickTime movies depicting solid black circles on a white background. Pilot data indicated that performance was at or near chance levels for the velocity judgments when typical tachistoscopic durations (i.e., $<200 \mathrm{msec}$ ) were employed; consequently, stimulus sequences of 300-msec duration were used.

Although this introduced the possibility that the subjects could make saccades and fixate the target before its offset, a number of considerations ameliorate this concern. First, Ross and Ross (1980) have presented data indicating that under conditions in which a fixation point remained constant preceding, during, and subsequent to target presentation (as in the present study), average saccade latency was between 280 and $290 \mathrm{msec}$. This suggests that, even if the subjects attempted to fixate the target on every trial, saccades would not have begun until, on average, over $90 \%$ of the stimulus presentation had been completed. Similarly, Saslow (1967) has reported that, under conditions comparable with those employed in the present study, only $2.5 \%$ of saccades had latencies under $240 \mathrm{msec}$, suggesting that the trials in the present study involved most, if not all, of the stimulus sequence's being viewed laterally. Although eye fixation was not explicitly monitored in the present study, the importance of maintaining central fixation was stressed to the subjects, and informal observation by the experimenter during practice trials indicated that the subjects complied with this request.

Over the course of the 300-msec stimulus presentation, a circle grew or shrank in size either quickly or slowly; quick versus slow size changes involved $1.0^{\circ}$ versus $0.5^{\circ}$ changes in diameter, respectively (i.e., $3.3^{\circ} \mathrm{sec}^{-1}$ vs. $1.65^{\circ} \mathrm{sec}^{-1}$ ). Initial and final circle sizes varied randomly so that judgments could not be made solely on the basis of initial or final size. Initial sizes ranged from $0.5^{\circ}$ to $1.5^{\circ}$, and final sizes ranged from $0.1^{\circ}$ to $1.9^{\circ}$; collapsed across all stimulus sequences, the average circle size was $1.0^{\circ}$. Stimuli were presented so that the center of the circle was located $3.1^{\circ}$ to the left or right of fixation.

Each movie consisted of a total of 11 frames presented at a rate of 30 frames $\sec ^{-1}$, with the first 10 frames constituting the actual stimulus presentation, and the 11th frame consisting of a blank frame to end stimulus presentation. The movies were generated by constructing individual frames (depicting dots of various sizes), using Aldus SuperPaint, v.3.0, which were saved as PICT files. These were then integrated into QuickTime movies using MooVer v.1.3, a shareware program. Stimulus presentation and response collection were under the control of MacLaboratory Reaction Time, v.3.0.2.

All the subjects participated in two tasks (both tasks were performed on the same stimulus set). The categorical task required the subjects to indicate whether the circle grew or shrunk in size (ignoring variations in velocity); the coordinate task required the subjects to indicate whether the circle changed size quickly or slowly (ignoring variations in direction of size change). Each task was run in a single block of 96 trials, yielded by the factorial combination 
of 2 visual fields $\times 2$ directions of motion $\times 2$ velocities $\times 12$ replications. Responses were made by pressing keys with the index fingers of their left and right hands; assignment of keys and task order were counterbalanced.

\section{Results and Discussion}

Two-way analyses of variance (ANOVAs), with task (categorical [grow/shrink] vs. coordinate [fast/slow]) and visual field (left visual field [LVF] vs. right visual field $[\mathrm{RVF}]$ ) as within-subjects factors, were performed on reaction time (RT) and error data (see Figure 1). For $\mathrm{RT}$, a main effect of task reflected faster responses in the categorical task $[F(1,15)=15.16, p=.001]$. No other effects were significant $\left(F_{\mathrm{S}}<1\right)$. Error data yielded a main effect of task, reflecting higher error rates for the coordinate task $(38.9 \%$, with $50 \%$ representing chance performance) than for the categorical task $[8.3 \% ; F(1,15)=$ $102.94, p=.001]$. A marginal effect of visual field reflected slightly greater accuracy in the $\operatorname{LVF}[F(1,15)=$ $3.39, p=.086]$. Finally, a significant interaction between task and visual field $[F(1,15)=3.39, p=.007]$ reflected no differences in accuracy for the categorical task, although there was a nominal RVF advantage $[F(1,15)=1.43, p=$ $.251]$ and a significant LVF advantage $[F(1,15)=7.35$, $p=.016]$ for the coordinate task.

Additional analyses were carried out on each task separately in order to examine effects of the specific judgment (i.e., grow vs. shrink, quick vs. slow). For the categorical task, there were no main effects or interactions for the RT data (all $p \mathrm{~s}>.32$ ); the only effect for the error data was a main effect of judgment $[F(1,15)=20.86, p=$ $.001]$, reflecting higher error rates for shrink judgments. For the coordinate task, the only significant effect for RT was an interaction between judgment and visual field $[F(1,15)=7.89, p=.013]$. This arose from the absence of visual field differences for quick judgments $[F(1,15)=$

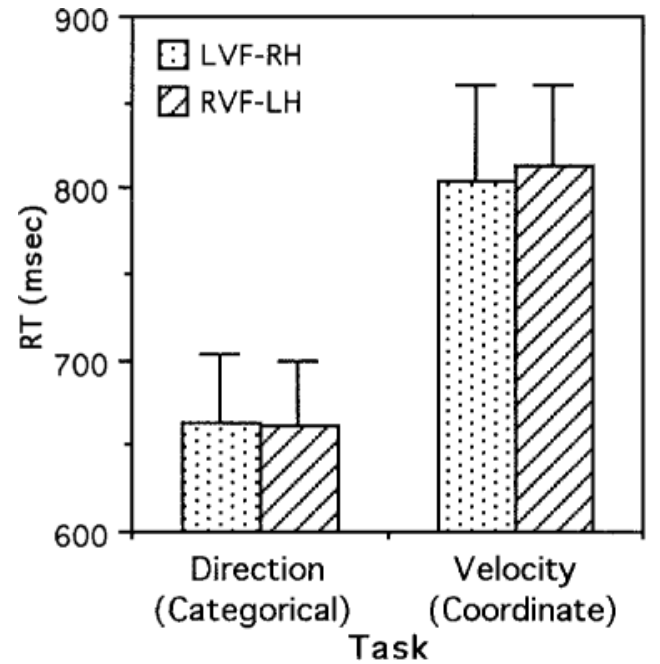

2.61, $p=.127]$ and a significant LVF advantage for slow responses $[F(1,15)=11.84, p=.004]$. For the error data from the coordinate task, the only significant result was a main effect of visual field $[F(1,15)=7.35, p=.016]$, indicating that the LVF advantage held equally for quick and slow judgments.

These results provide initial support for an extension of Kosslyn's (1987) hypothesis of hemispheric differences in the processing of categorical versus coordinate spatial relations to the processing of dynamic visual input. Coordinate judgments requiring subjects to make precise judgments about the rate of change in size yielded a significant LVF advantage in accuracy. Categorical judgments requiring subjects to make dichotomous judgments about direction of change (i.e., shrink or grow) yielded nominal RVF advantages. Although significant hemispheric differences for the categorical task were not obtained, previous studies have also often revealed no significant hemispheric differences on categorical tasks. Kosslyn et al. (1992) noted, however, that although LH categorical advantages are rarely significant in individual studies, a nonparametric meta-analysis indicated that there does seem to be a real (albeit small) $\mathrm{LH}$ advantage for categorical processing.

\section{EXPERIMENT 2}

The purpose of Experiment 2 was to extend the findings of Experiment 1 to a different type of motion judgment. Namely, whereas Experiment 1 employed changes in the size of a circle, Experiment 2 employed changes in the position of a circle, which moved upward or downward at either a fast or a slow velocity. Up/down judgments constituted the categorical task, whereas quick/slow judgments constituted the coordinate task.

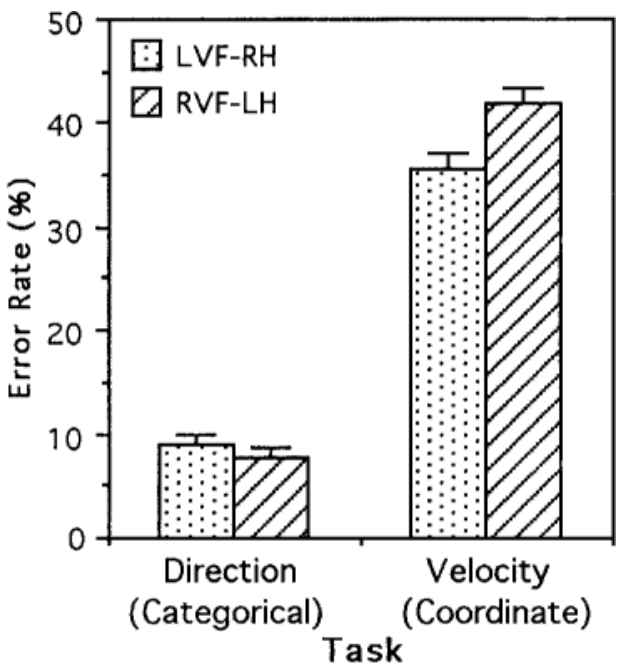

Figure 1. Reaction times (RTs) and error rates as a function of visual field and task (categorical, direction of motion; coordinate, velocity of motion) for Experiment 1. Error bars indicate standard error values. LVF, left visual field; RVF, right visual field; RH, right hemisphere; LH, left hemisphere. 


\section{Method}

Subjects. Sixteen right-handed subjects with no left-handed relatives in their immediate family participated in the study in order to receive extra credit in an introductory psychology course. Handedness was assessed via use of the Edinburgh Handedness Inventory (Oldfield, 1971).

Apparatus and Procedure. Details of stimulus preparation and presentation were the same as those in Experiment 1, with the following exceptions. Over the course of the 300 -msec stimulus presentation, a solid circle of $1.0^{\circ}$ diameter moved vertically upward or downward with a fast or a slow velocity. The dot moved a total of $1.0^{\circ}$ (equivalent to a velocity of $3.3^{\circ} \mathrm{sec}^{-1}$ ) in the fast velocity condition and $0.5^{\circ}$ (equivalent to a velocity of $1.65^{\circ} \mathrm{sec}^{-1}$ ) in the slow velocity condition. Initial and final dot positions were varied randomly across a $1.0^{\circ}$ range in order to prevent the subjects from using initial or final position as a potential cue.

All the subjects participated in two tasks (both tasks were performed on the same stimulus set). The categorical task required the subjects to indicate whether the circle moved upward or downward; the coordinate task required the subjects to indicate whether the circle changed position quickly or slowly. Each task was run in a single block of 48 trials, yielded by the factorial combination of 2 visual fields $\times$ 2 directions of motion $\times 2$ velocities $\times 6$ replications. Responses were made by pressing keys with the index fingers of their left and right hands; assignment of keys and tasks was counterbalanced.

\section{Results and Discussion}

Two-way ANOVAs, with task (categorical [upward/ downward] vs. coordinate [fast/slow]) and visual field (LVF vs. RVF) as within-subjects factors, were performed on RT and error data (see Figure 2). For RT, the only significant effect was the interaction between task and visual field $[F(1,15)=4.65, p=.048]$. Analyses of simple effects revealed that this interaction arose from the presence of no visual field differences for the categorical task $(F<1)$ and the presence of a significant LVF advantage for the coordinate task $[F(1,15)=4.78, p=$ .045]. Additional analyses for each task separately yielded no significant effects of judgment type (up vs.

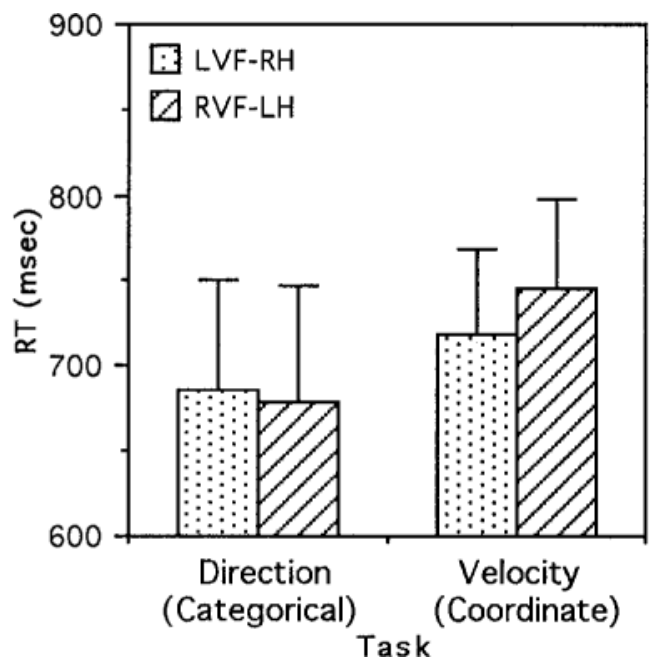

down for the categorical task; fast vs. slow for the coordinate task; all $p \mathrm{~s}>.311$ ).

For error data, the only significant effect was for task $[F(1,15)=19.35, p=.001]$, reflecting higher error rates for the coordinate task. Additional analyses for each task separately yielded no effects of judgment type for either task (all $p$ s > .191).

These results echo the results of Experiment 1: Although no visual field differences were found for the categorical task, a significant LVF advantage in RT was obtained for the coordinate task. Although the significant LVF advantage in Experiment 1 was obtained for error rates, and not for RTs, this likely reflected the fact that the task in Experiment 1 was more difficult, yielding higher RTs and error rates. The lack of visual field differences for the categorical task again mirrored a general trend in research for categorical tasks to yield nonsignificant or marginal visual field differences.

\section{EXPERIMENT 3}

Although Experiments 1 and 2 provide moderately strong support for the extension of Kosslyn's (1987) categorical/coordinate framework to hemispheric differences in motion perception, the fact that exposure durations in those experiments were long enough to allow the possibility of eye movements and foveal fixation of stimuli on at least some trials leaves open the possibility that the results may have reflected oculomotor biases, and not true hemispheric differences. For example, perhaps the subjects were able in the coordinate tasks to move their eyes more quickly and regularly to the left, thereby fixating LVF stimuli and leading to LVF advantages that reflected better acuity rather than an RH superiority for the task itself. Although this is unlikely, since evidence suggests that saccadic latency is shorter for rightward than

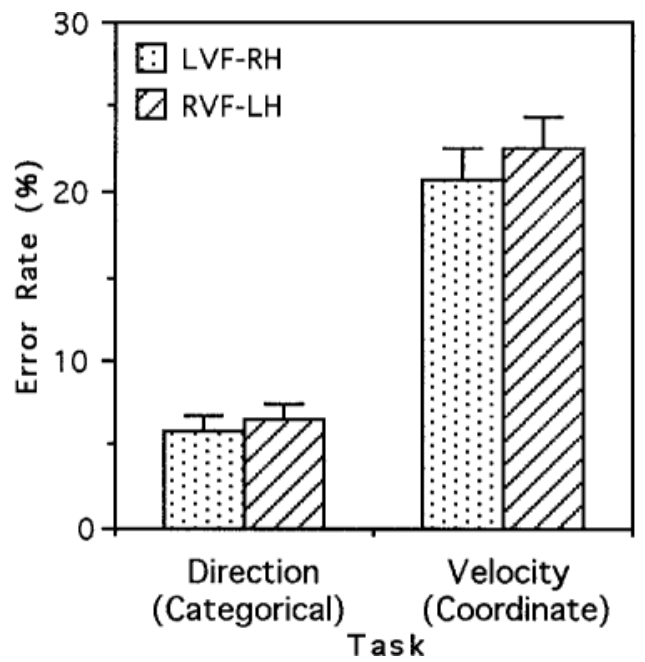

Figure 2. Reaction times (RTs) and error rates as a function of visual field and task (categorical, direction of motion; coordinate, velocity of motion) for Experiment 2. Error bars indicate standard error values. LVF, left visual field; RVF, right visual field; RH, right hemisphere; LH, left hemisphere. 
for leftward eye movements (Hutton \& Pallet, 1986), a final experiment was conducted, employing the same general tasks as in Experiment 2 but employing a shorter exposure duration of $150 \mathrm{msec}$ to ensure lack of eye movements during stimulus presentation.

\section{Method}

Subjects. Sixteen right-handed subjects with no left-handed relatives in their immediate family participated in the study in order to receive extra credit in an introductory psychology course. Handedness was assessed via use of the Edinburgh Handedness Inventory (Oldfield, 1971).

Apparatus and Procedure. Details of stimulus preparation, presentation, and procedure were the same those as in Experiment 2, with the following change. In order to employ a shorter exposure duration, stimuli were reduced down to five frames presented at $30 \mathrm{msec}$ per frame, yielding a duration of $150 \mathrm{msec}$. Because pilot data utilizing this duration and the velocities utilized in Experiment 2 indicated that performance on the coordinate task was at or near chance, the velocities were doubled, so that fast versus slow velocities corresponded to $6.6^{\circ} \mathrm{sec}^{-1}$ versus $3.3^{\circ} \mathrm{sec}^{-1}$.

\section{Results and Discussion}

Two-way ANOVAs, with task (categorical [upward/ downward] vs. coordinate [fast/slow]) and visual field (LVF vs. RVF) as within-subjects factors, were performed on RT and error data (see Figure 3). For RT, a significant main effect of task $[F(1,15)=12.96, p=.003]$ reflected faster RTs for the categorical task. There was also a significant task $\times$ visual field interaction $[F(1,15)=6.10$, $p=.026]$. Analyses of each task separately revealed no visual field differences in the categorical task $(p=.182)$ and a significant LVF advantage for the coordinate task $[F(1,15)=4.55, p=.050]$. There were no main effects or interactions involving judgment type for either task.

Analyses of the error data yielded a significant main effect of task $[F(1,15)=33.22, p=.001]$, reflecting higher error rates in the coordinate task. There was a marginal trend toward a task $\times$ visual field interaction $[F(1,15)=3.48, p=.082]$. Analyses of simple effects were conducted for each task separately, with visual field and judgment type as within-subjects factors. For the categorical task, there was a significant main effect of judgment $[F(1,15)=12.91, p=.003]$ and a marginal main effect of visual field $[F(1,15)=4.11, p=.061]$; both of these effects were qualified by a significant interaction between visual field and judgment type $[F(1,15)=7.49, p=.015]$. This interaction arose from the fact that upward judgments yielded a significant RVF advantage $[F(1,15)=7.14, p=.017]$, whereas downward judgments yielded a nonsignificant LVF advantage $[F(1,15)=3.17, p=.095]$. For the coordinate task, there were no main effects or interactions.

The present results again yielded a task $\times$ visual field interaction consistent with Experiments 1 and 2. An LVF advantage was found for RT in the coordinate task, and an RVF advantage for accuracy was found in the categorical task, particularly for judgments of upward motion.

\section{GENERAL DISCUSSION}

Across all three experiments, visual field differences in motion perception were obtained. Furthermore, these differences were dependent on the aspect of motion being judged. For categorical judgments of the direction of motion, hemispheric differences were generally absent, although an RVF/LH advantage in accuracy was obtained in Experiment 3. Although there were nominal RVF advantages in RT for categorical judgments of direction of motion across all three experiments and in accuracy in Experiments 1 and 3, these advantages did not obtain significance even in a post hoc analysis combining data across all three experiments (LVF RT $=644 \mathrm{msec}$, RVF $\mathrm{RT}=634 \mathrm{msec}, p=.234$; $\mathrm{LVF}$ errors $=3.40, \mathrm{RVF}$ errors $=$

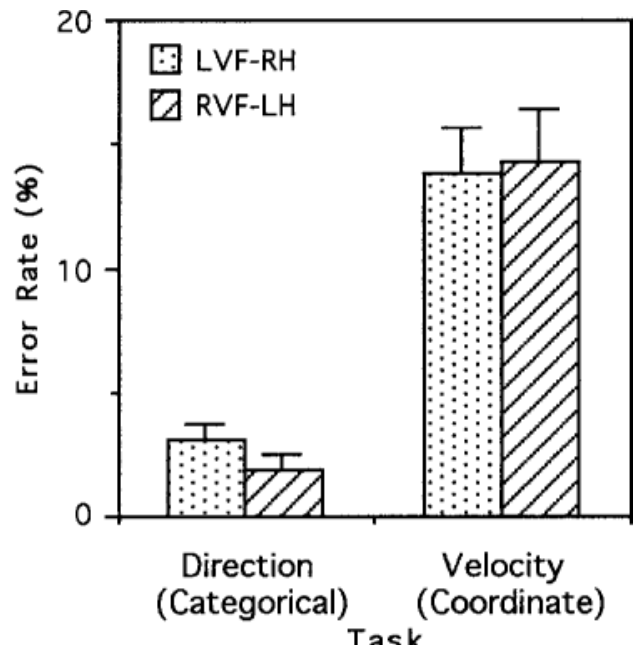
Task

Figure 3. Reaction times (RTs) and error rates as a function of visual field and task (categorical, direction of motion; coordinate, velocity of motion) for Experiment 3. Error bars indicate standard error values. LVF, left visual field; RVF, right visual field; RH, right hemisphere; LH, left hemisphere. 
$2.88, p=.093$ ). For coordinate judgments of the velocity of motion, on the other hand, significant RH advantages were found across all three experiments (for accuracy in Experiment 1 and for RT in Experiments 2 and 3).

The fact that the coordinate task yielded robust and reliable visual field differences, whereas the categorical task did not, is consistent with previous research on the categorical-coordinate processing distinction (e.g., Kosslyn et al., 1992). Although the reason for this pattern of results is not clear, Kosslyn et al. (1992) suggested that the $\mathrm{LH}$ advantage in categorical processing is attenuated or eliminated in situations in which it is difficult to compute a spatial relation by defining discrete pockets of space (e.g., space above vs. below a line). Although this is readily done with the line-dot stimuli discussed above, it is not clear how moving stimuli would allow a strict, categorical delineation of space to define upward versus downward motion, especially since the starting and ending points of the stimuli in the present experiments were randomized. Importantly, however, all three experiments yielded significant task $X$ visual field interactions, demonstrating a true hemispheric dissociation between the two types of motion judgments.

The fact that coordinate judgments, which yielded consistent RH advantages, were also consistently slower and less accurate than categorical judgments raises the possibility that the hemispheric differences obtained in the present study reflect variations in task difficulty, instead of type of motion judgment. However, other perceptual variables that have been shown to yield hemispheric differences display the opposite pattern. For example, it is well established that the $\mathrm{RH}$ and the $\mathrm{LH}$ are specialized for the processing of low versus high spatial frequencies, respectively (e.g., Christman, 1997b). However, in this case, the more difficult judgments involve higher spatial frequencies, which yield LH advantages. Thus, there is little reason to think that the present results simply reflect a general RH advantage for difficult tasks.

It is worth considering how the present results relate to prior studies of motion perception in the hemispheres. Generally, prior research has utilized only a single type of judgment. Some studies have employed tasks involving judgments of direction of motion, roughly akin to the categorical judgments employed in the present study. Kostelyanets, Kamenkovich, Shevelev, and Sharaev (1989) reported LH superiority in identification of direction of motion. Kostelyanets et al. (1992) also concluded that the $\mathrm{LH}$ is superior at detection of direction of motion. Hollants-Gilhuijs et al. (1998) reported no hemispheric difference in adults in processing direction of motion, although children exhibited an LH advantage. Bosworth and Dobkins (1999) reported a slight RH advantage for identification of direction of motion in hearing subjects (deaf subjects showed a strong LH advantage). Thus, somewhat in line with the present results, there do not seem to be pronounced or consistent hemispheric asymmetries in processing direction of motion, although the work of Kostelyanets and colleagues suggests a possible LH superiority.

Other studies have involved judgments focusing on velocity of motion, akin to the coordinate judgments employed in the present study. Smith and Hammond (1986) employed a velocity-matching paradigm and found no overall hemispheric differences (although individual subjects typically displayed robust asymmetries). Eals (1987) presented observers with two successive frames that induced apparent motion; observers then had to choose a third frame from an array of choices that represented possible extrapolations of the motion induced by the first two. The limited information provided about the nature of the stimuli suggests that the extrapolated distances of the dots' motions were critical for accurate performance. The RH advantage reported by Eals might thus be consistent with the present findings of $\mathrm{RH}$ advantages for velocity judgments, since judgments of inferred distance rely on estimates of the stimulus' apparent velocity. Regan and Vincent (1995) reported no hemispheric differences in judgments of time to contact and rate of expansion (the use of monocular viewing in this study, however, obscures the relative contribution of hemispheric vs. hemiretinal effects). Thus, these studies are somewhat consistent with the present findings, since judgments of velocity yielded either RH superiority or no hemispheric differences.

Finally, brain imaging studies, in which the experimenter has little control over how observers treat the stimuli, have yielded results suggesting greater RH involvement (Dieterich et al., 1998; Hollants-Gilhuijset al., 2000).

At the very least, the present findings indicate that it is unlikely that a single hemisphere is specialized for all aspects of motion perception; rather, the ability of the $\mathrm{LH}$ versus the RH to extract information about motion appears to depend on the specific type of information being extracted. Extraction of information related to categorical aspects of motion (i.e., direction) seems to be performed roughly equivalently in the two hemispheres (although there may be a slight LH advantage). Extraction of coordinate information involving precise aspects of motion (i.e., velocity), on the other hand, is performed more efficiently in the RH. Thus, future research on visual field differences (both left-right and upper-lower) in motion perception should consider utilizing different types of motion judgments in order to determine the extent to which visual field differences are task dependent.

An important caveat needs to be made concerning these conclusions-namely, that motion judgments of the sort employed in the present study can potentially be based on two distinct types of process: motion sensitivity per se versus position sensitivity, based on comparisons of initial and final positions (e.g., Nakayama \& Tyler, 1981). That is, performance on the present tasks could be based on the explicit processing of motion, or it could be based on a special case of the categorical/coordinate spatial processing proposed by Kosslyn (1987), 
in which the positions of two stimuli (e.g., initial and final dot positions) are compared successively across time, instead of simultaneously (as in the line-dot stimuli discussed above). Recent work by Seiffert and Cavanagh $(1998,1999)$ indicates that the position-sensitive system operates preferentially on low-contrast, slowly moving (e.g., less than $1^{\circ} \mathrm{sec}^{-1}$ ) stimuli, whereas the velocity-sensitive mechanism operates preferentially on high-contrast, quickly moving stimuli. Given the high contrasts and velocities used in the present experiments, this suggests that performance might have been based on the velocity-sensitivemechanism. Definitive resolution of this question, however, would require the use of randomdot kinematograms (e.g., Braddick, 1974) or stochastic motion displays (e.g., Newsome \& Pare, 1988), for which motion judgments cannot be based on high-level positional tracking mechanisms.

Finally, the present experiments extend Kosslyn's (1987) categorical-coordinate framework. This theory was originally applied to the extraction of spatial relations between static, simultaneously present stimuli; the present results demonstrate that this framework applies also to the spatial relations between successive views of a single moving stimulus. Thus, the present study adds to the growing literature on extensions of the categoricalcoordinate framework. For example, David and Cutting (1992) extended it to conceptual judgments; categorical judgments regarding a pictorial stimulus's status as living versus nonliving produced an LH advantage, whereas judgments of size yielded an RH advantage. Wiener and Christman (1996) extended the framework to facial processing, showing that categorical judgments of gender yielded an LH advantage, whereas coordinate judgments of a face's specific identity yielded RH advantages. Niebauer and Christman (1999) utilized a spatial frequency discrimination task, arguing that conditions in which frequency differences were large could be readily categorized as thick versus thin, whereas conditions in which the frequency difference was small would require coordinate judgments of the exact width of the bars of the gratings. Consistent with these assumptions, they found a significant RH advantage in the coordinate task, but no differences in the categorical task. Finally, Wiener and Christman (1994) extended the categorical-coordinate framework to judgments of auditory tonal relations between two successively presented tones; categorical judgments of whether the second tone was above or below the pitch of the first tone yielded right-ear-LH advantages, whereas coordinate judgments of whether the second tone was near or far in pitch from the first one yielded left-ear-RH advantages.

It thus appears that the hypothesis that the $\mathrm{LH}$ and the $\mathrm{RH}$ are specialized for the extraction of categorical versus coordinate stimulus relations has a wide range of applicability, including judgments of both static and dynamic spatial relations, judgments of conceptual attributes, and judgments performed on such diverse stimuli as sinusoidal gratings, photographs of faces, and audi- tory tones. In addition, researchers interested in motion processing, but not brain asymmetry per se, should pay attention to the types of information underlying different judgments of motion, since the existence of different neural substrates for such judgments presumably has functional implications, even under conditions of nonlateralized stimulus presentation.

\section{REFERENCES}

Bosworth, R., \& Dobkins, K. (1999). Left hemisphere dominance for motion processing in deaf signers. Psychological Science, 10, 256262.

BRADDICK, O. (1974). A short-range process in apparent motion. Vision Research, 14, 519-527.

Calvert, J. (1988). Adaptation to movement in right and left visual fields. Cortex, 24, 589-593.

Christman, S. (ED.) (1997a). Cerebral asymmetries in sensory and perceptual processing. Amsterdam: North-Holland.

Christman, S. (1997b). Hemispheric asymmetry in the processing of spatial frequency: Experiments using gratings and bandpass filtering. In S. Christman (Ed.), Cerebral asymmetries in sensory and perceptual processing (pp. 3-30). Amsterdam: North-Holland.

DAvid, A. S., \& CutTing, J. C. (1992). Categorical-semantic and spatialimagery judgments of non-verbal stimuli in the cerebral hemispheres. Cortex, 28, 39-51.

De RenZi, E. (1982). Disorders of space exploration and cognition. New York: Wiley.

Dieterich, M., Bucher, S., Seelos, K., \& Brandt, T. (1998). Horizontal or vertical optokinetic stimulation activates visual motionsensitive, ocular motor and vestibular cortex areas with right hemispheric dominance: An fMRI study. Brain, 121, 1479-1495.

EALS, M. (1987). Asymmetric processing in perception of apparent movement. Neuropsychologia, 25, 429-434.

Hellige, J. B., \& Michimata, C. (1989). Categorization versus distance: Hemispheric differences for processing spatial information. Memory \& Cognition, 17, 770-776.

Hollants-Gilhuiss, M., De Munck, J., Kubova, Z., Royen, E., \& SPEKREIJSE, H. (2000). The development of hemispheric asymmetry in human motion VEPs. Vision Research, 40, 1-11.

Hollants-Gilhuiss, M., Ruijter, J., \& Spekreijse, H. (1998). Visual half-field development in children: Detection of motion-defined forms. Vision Research, 38, 651-657.

Hutton, J., \& Pallet, J. (1986). Lateral saccadic latencies and handedness. Neuropsychologia, 24, 449-451.

Kitterle, F. L., Christ man, S., \& Hellige, J. B. (1990). Hemispheric differences are found in the identification, but not the detection, of low versus high spatial frequencies. Perception \& Psychophysics, 48, 297-306.

Kosslyn, S. M. (1987). Seeing and imaging in the cerebral hemispheres: A computational approach. Psychological Review, 94, 148-175.

Kosslyn, S. M., Chabris, C. F., Marsolek, C., \& Koenig, O. (1992). Categorical versus coordinate spatial relations: Computational analyses and computer simulations. Journal of Experimental Psychology: Human Perception \& Performance, 18, 562-577.

Kosslyn, S. M., Koenig, O., Barrett, A., Cave, C. V., Tang, J., \& Gabrieli, J. E. E. (1989). Evidence for two types of spatial representations: Hemispheric specialization for categorical and coordinate relations. Journal of Experimental Psychology: Human Perception \& Performance, 15, 723-735.

Kostelyanets, N., Kamenkovich, V., \& Sharaev, G. (1992). Two mechanisms of recognition of the direction of motion in humans. Sensory Systems, 6, 59-63.

Kostelyanets, N., Kamenkovich, V., Shevelev, I. A., \& Sharaev, G. (1989). Interhemispheric asymmetry in visual perception of motion. Sensory Systems, 3, 231-234.

Nakayama, K., \& Tyler, C. W. (1981). Psychophysical isolation of movement sensitivity by removal of familiar position cues. Vision Research, 21, 427-433. 
Newsome, W. T., \& PAre, E. B. (1988). A selective impairment of motion perception following lesions of the middle temporal visual area (MT). Journal of Neuroscience, 8, 2201-2211.

Niebauer, C. L., \& Christman, S. D. (1998). Upper and lower visual field differences in categorical and coordinate judgments. Psychonomic Bulletin \& Review, 5, 147-151.

Niebauer, C. L., \& Christman, S. D. (1999). Visual field differences in spatial frequency discrimination. Brain \& Cognition, 41, 381-389.

OldFIELD, R. (1971). The assessment and analysis of handedness: The Edinburgh Inventory. Neuropsychologia, 9, 97-113.

Regan, D., \& VIncent, A. (1995). Visual processing of looming and time to contact throughout the visual field. Vision Research, 35, 1845-1857.

Ross, L. E., \& Ross, S. M. (1980). Saccade latency and warning signals: Stimulus onset, offset, and change as warning events. Perception \& Psychophysics, 27, 251-257.

SASLOW, M. (1967). Effects of components of displacement-step stimuli upon latency for saccadic eye movement. Journal of the Optical Society of America, 57, 1024-1029.
Seiffert, A. E., \& Cavanagh, P. (1998). Position displacement, not velocity, is the cue to motion detection of second-order stimuli. Vision Research, 38, 3569-3582.

Seiffert, A. E., \& Cavanagh, P. (1999). Position-based motion perception for color and texture stimuli: Effects of contrast and speed. Vision Research, 39, 4172-4185.

Smith, A. T., \& Hammond, P. (1986). Hemifield differences in perceived velocity. Perception, 15, 111-117.

Wiener, R, \& Christman, S. (1994, November). Hemispheric asymmetry in the processing of categorical versus coordinate auditory pitch relations. Paper presented at the 35th Annual Meeting of the Psychonomic Society, St. Louis.

Wiener, R, \& Christman, S. (1996, February). Categorical and coordinate processing of facial stimuli. Presented at the Annual Meeting of the International Neuropsychological Society, Chicago.

(Manuscript received November 9, 2000; revision accepted for publication June 1, 2001.) 